\title{
Benefit analysis and evaluation of virtual power plants considering electric vehicles
}

\author{
Tan Caixia ${ }^{1, *}$, Tan Zhongfu' ${ }^{1}$, Wu Jianbin², Qi Huiwen², Zhang Xiangyu ${ }^{2}$ and Xu Zhenbo ${ }^{2}$ \\ ${ }^{1}$ School of Economics and Management, North China Electric Power University, 102206 Beijing, China \\ ${ }^{2}$ Economic and Technical Research Institute of Shanxi Electric Power Company, State Grid, 030000 Taiyuan, Shanxi Province, China
}

\begin{abstract}
Under the background of increasingly serious environmental pollution, virtual power plants have become an effective way to solve environmental pollution due to the characteristics of integrating a large number of clean distributed energy generation. At the same time, electric vehicles with dual attributes of power supply and load bring opportunities for the further development of virtual power plants. In this paper, the ideal matter-element comprehensive benefit evaluation model of virtual power plants is constructed by constructing the index system and weighting model of virtual power plants considering electric vehicles. The virtual power plants connected with different proportion of electric vehicles are taken as an example analysis. The results of the example analysis show that the comprehensive benefit of virtual power plants is the highest when the proportion of electric vehicles access reaches $120 \%$.
\end{abstract}

\section{Introduction}

With the increasingly prominent environmental problems, virtual power plants with clean and renewable energy can effectively solve the problem of environmental pollution. Due to the uncertainty of renewable energy output, electric vehicles with dual attributes of power supply and load can effectively solve this problem. Therefore, the virtual power plants considering electric vehicles has become a research hotspot, especially about the benefit analysis and evaluation of virtual power plants considering electric vehicles.

There are little research on the evaluation of virtual power plants. Based on the potential game, the authors of 1 proposed a comprehensive utility calculation method considering the comprehensive efficiency and economic benefits of enterprises, and constructed a member selection model of virtual power plants for fire-windstorage high load energy enterprises. The authors of 2 provided a comprehensive evaluation index system with perfect hierarchy and structure for thermal power plants. The first level indexes were divided into four categories: reliability, economy, technical supervision and main operation; The authors of 4 provided a comprehensive evaluation index system composed of seven indexes from three aspects of reliability, economy and schedulability to evaluate the effect of virtual power plants participating in active power dispatching of power grid; The authors of 6 proposed the target comprehensive evaluation technology based on the relevant research of virtual power plant active power dispatching, including index system and evaluation method. The current research has evaluated the scheduling optimization effect of virtual power plants from multi-dimensional, but there is little research on the benefit analysis and evaluation of virtual power plants considering electric vehicles.

Based on the existing research, this paper constructs the evaluation index system of virtual power plants from three aspects of economic benefit, environmental benefit and social benefit, and evaluates the virtual power plant by using the ideal matter-element extension model. Finally, four kinds of virtual power plants are taken as the evaluation schemes for example analysis.

\section{Construction of index system and index weighting model of virtual power plants considering electric vehicles}

\subsection{Construction of index system}

Based on the economic benefit, environmental benefit and social benefit, the evaluation index system of virtual power plants considering electric vehicles is established, which includes three first level indexes, seven second level indexes and 24 third level indexes, which is shown in Table 1:

Table 1. Basic parameters of gas turbine.

\begin{tabular}{|c|c|c|}
\hline $\begin{array}{c}\text { Economic } \\
\text { benefit } \\
\text { A1 }\end{array}$ & $\begin{array}{c}\text { Generation } \\
\text { side benefit } \\
\text { B1 }\end{array}$ & $\begin{array}{c}\text { Avoiding capacity cost of peak } \\
\text { load C1 } \\
\text { Avoiding fuel cost of power } \\
\text { generation C2 } \\
\text { Avoiding cost of units start-up } \\
\text { and shutdown C3 } \\
\text { Avoiding cost of power } \\
\text { construction C4 }\end{array}$ \\
\hline
\end{tabular}

* Corresponding author: 2858877150·@qq.com 


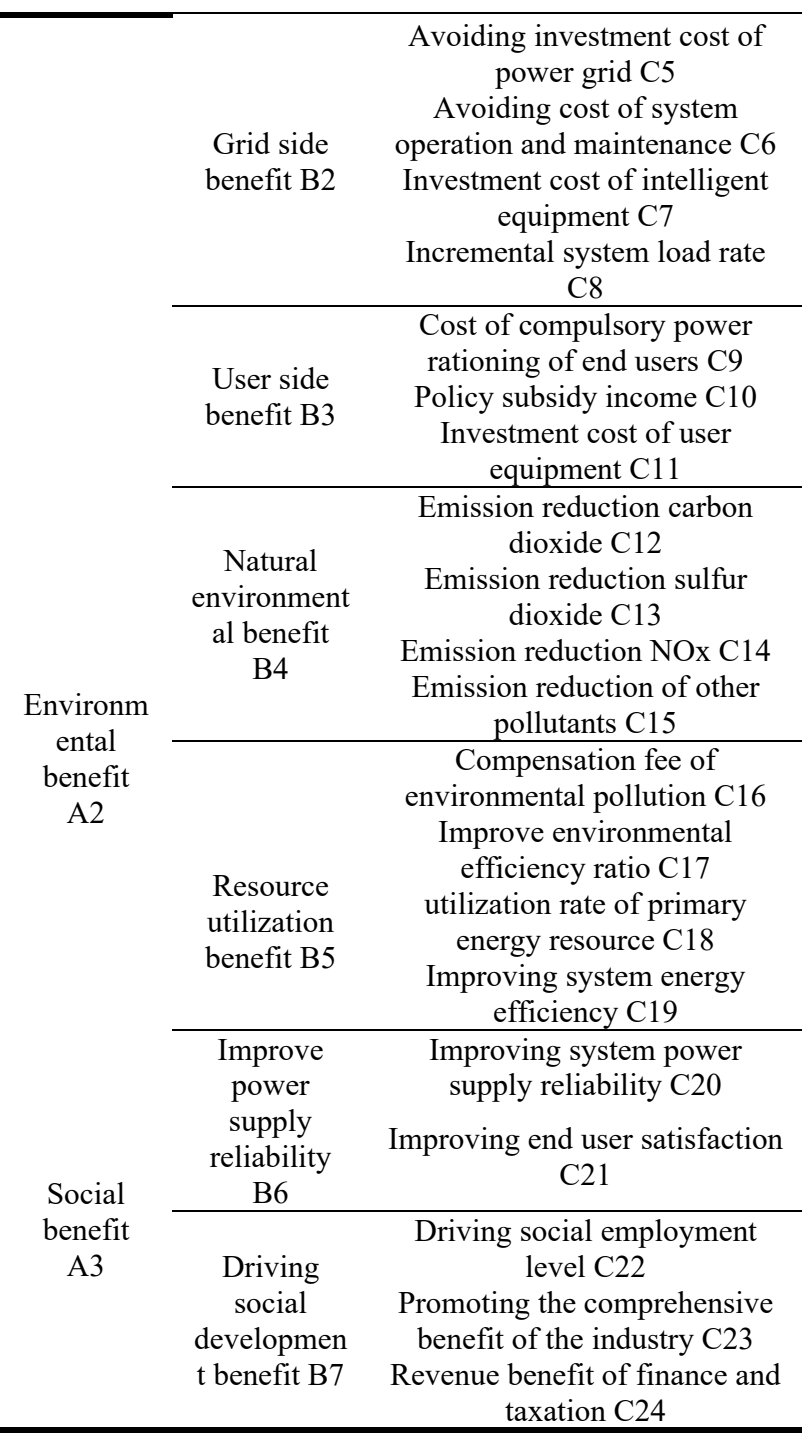

Because the benefit evaluation index system of electric vehicles accessing to virtual power plants has the characteristics of multi-level and multi-index, in order to facilitate comparative analysis, it is necessary to eliminate the difference of unit dimension of evaluation index. At the same time, the evaluation index has positive index, negative index and intermediate index. Before the evaluation, all kinds of evaluation indexes need to be consistent. The specific treatment methods are as follows:

For the positive index, the processing is shown in equation (1)

$$
a_{i j}=\frac{p_{i j}-m_{j}}{M_{j}-m_{j}}
$$

For the negative index, the processing is shown in equation (2)

$$
a_{i j}=\frac{M_{j}-P_{i j}}{M_{j}-m_{j}}
$$

Where $M_{j}=\max _{i}\left\{p_{i j}\right\}, m_{j}=\min _{i}\left\{p_{i j}\right\}$. After the transformation, the maximum value of the index is 1 and the minimum value is 0 .

For the intermediate index, the processing is shown in equation (3).

$$
\mathrm{A}(p)=\left\{\begin{array}{cc}
0 & p<a \\
(p-a) /(b-a) & a \leq p \leq b \\
1 & b \leq p \leq c \\
(d-p) /(d-c) & c \leq p \leq d \\
0 & d \leq p
\end{array}\right.
$$

Where $a, d$ represent the lower limit and upper limit of the function respectively; $b, c$ represent the two ends of the moderate interval respectively.

\subsection{Index weighting model}

The selection of weighting method and evaluation method will directly affect the evaluation results in benefit evaluation. As for the weighting method, it is mainly divided into subjective weighting method and objective weighting method. The subjective weighting method mainly depends on expert knowledge and experience to judge the subjective importance of each index. The main subjective weighting method include eigenvalue method, order relation method and scheme preference weighting method. Objective weighting method is based on the original data, using more perfect mathematical theory and method to determine the index weight. The main objective weighting method include entropy weighting method, rough set method, etc. Subjective weighting method reflects the subjective judgment and intuition of the evaluator, but the evaluation results may produce some subjective randomness. Objective weighting method avoids the subjectivity of the evaluation results, but ignores the subjective information necessary for safety evaluation. Therefore, ANP method and entropy weighting method are used to weight the index in this paper.

(1) ANP method

Construction of ANP hypermatrix. Let $P_{1}, \ldots, P_{n}$ be in the control layer of ANP, and $C_{1}, \ldots, C_{n}$ be in the network layer below the control layer, where $e_{i 1}, \ldots, e_{i n}$, $i=1, \ldots, N$ is in $C_{i}$. Taking control layer element $P_{s}(s=1, \ldots, m)$ as the criterion and element $e_{j 1}$ in $C_{j}$ as the criterion, the elements in element group $C_{i}$ are compared in indirect dominance according to their influence on $e_{j 1}$, that is, the judgment matrix is constructed. ANP model is scaled by the nine-point method, and the ranking vector is obtained by the eigenvalue method. If the consistency test is satisfied, the above eigenvector will be the ranking vector (weight) of network elements, and all the ranking vectors of network elements are combined and constructed as a matrix:

$$
W_{i j}=\left[\begin{array}{cccc}
w_{i 1}^{j 1} & w_{i 1}^{j 2} & \cdots & w_{i 1}^{j n_{j}} \\
w_{i 2}^{j 1} & w_{i 2}^{j 2} & \cdots & w_{i 2}^{j n_{j}} \\
\cdots & \cdots & \cdots & \cdots \\
w_{i n_{j}}^{j 1} & w_{i n_{j}}^{j 2} & \cdots & w_{i i_{j}}^{j n_{j}}
\end{array}\right]
$$

The column vector of $W_{i j}$ represents the importance 
ranking vector of element $C_{i}$ to element $C_{j}$. By combining the order vectors of all the elements in the network layer, the hypermatrix under the control element is obtained:

$$
\left.\begin{array}{rr|rrrr}
1 & W_{11} & W_{12} & \cdots & W_{1 N} \\
\cdots & & & & \\
n_{1} & & & & \\
1 & & & & \\
\cdots= & W_{21} & W_{22} & \cdots & W_{2 N} \\
n_{2} & & & & \\
\cdots & & & & \\
1 & & & & \\
\cdots & & & & \\
n_{N} & W_{N 1} & W_{N 2} & & W_{N 3}
\end{array}\right]
$$

Every element of the matrix is a matrix and the sum is 1 , but it is not the normalized matrix. In order to calculate conveniently, it is necessary to normalize the hypermatrix, that is, to weight the elements of the hypermatrix to get the weighted hypermatrix $\bar{W}=(\bar{W})_{n \times n}, \bar{W}_{i j}=a_{i j} W_{i j}$, and $a_{i j}$ is the weighting factor, $i, j=1,2, \ldots, n$.

In order to reflect the dependency relationship between elements, it is necessary to make a stable output for the hypermatrix, that is, to calculate the limit relative ordering vector of each hypermatrix:

$$
\lim (1 / N) \sum_{k \rightarrow \infty}^{N} \bar{W}^{k}
$$

If the above limit is convergent and unique, the value of the corresponding row of the element matrix is the stable weight of each evaluation index.

(2) Entropy weighting method

Suppose that the different integration modes of virtual power plants benefit are $a=\left\{a_{1}, a_{2}, \ldots, a_{n}\right\}$, and the comprehensive evaluation index system of each integration mode is $u=\left\{u_{1}, u_{2}, \ldots, u_{n}\right\}$. If the attribute value of integration mode $a_{i}(i=1,2, \ldots, m)$ under index $u_{j}(j=1,2, \ldots, n)$ is $a_{i j}$, then the decision matrix is $A=\left(a_{i j}\right)_{m \times n}$, denote $M=(1,2, \ldots, m)$ and $N=(1,2, \ldots, n)$. Generally, the types of indicators are generally benefit type and cost type. Because the dimensions of different attributes may be different, in order to eliminate the impact of different dimensions on the decision-making results, it is necessary to treat the attribute index as dimensionless.

For the attributes of benefit type, the processing is shown in equation (7)

$$
r_{i j}=\frac{a_{i j}-\min _{i} a_{i j}}{\max _{i} a_{i j}-\min _{i} a_{i j}}
$$

For the attributes of cost type, the processing is shown in equation (8)

$$
r_{i j}=\frac{\max _{i} a_{i j}-a_{i j}}{\max _{i} a_{i j}-\min _{i} a_{i j}}
$$

The matrix $R=\left(r_{i j}\right)_{m \times n}$ obtained by the above dimensionless processing is called the normalized decision matrix.

First, the entropy $E_{j}$ of attribute index $j$ is calculated

$$
E_{j}=-k \sum_{i=1}^{n} r_{i j} \ln \left(r_{i j}\right), j=1,2, \ldots, m
$$

Where $j=1,2, \ldots, m, k=1 / \ln (n)$ is a constant related to the number of samples, so that $r_{i j}$ satisfies $0<r_{i j}<1$ and $\sum_{i=1}^{n} r_{i j}=1$, and when $r_{i j}=0, r_{i j} \ln \left(r_{i j}\right)=0$.

Then, the information deviation degree is calculated as shown in Equation (10)

$$
d_{j}=1-E_{j}
$$

Finally, the index weight is calculated as shown in Equation (11)

$$
w_{j}=\frac{d_{j}}{\sum_{j=1}^{m} d_{j}}=\frac{1-E_{j}}{m-\sum_{j=1}^{m} E_{j}},(j=1,2, \ldots, m)
$$

(3) Subjective and objective integrated weighting model

In order to overcome the shortcomings of subjective and objective weighting method and make the index weighting take into account both subjective and objective factors, this section constructs a comprehensive integrated weighting model with the objective of minimizing the deviation degree of subjective and objective weights to determine the weight of the evaluation indexes.

Suppose that the subjective weight vector of the evaluation index of virtual power plants calculated by ANP method is $w^{\prime}=\left(w_{1}^{\prime}, w_{2}^{\prime}, \ldots, w_{n}^{\prime}\right)^{T}$ which satisfis $w_{j}^{\prime} \in[0,1], \quad \sum_{j=1} w_{j}^{\prime}=1$. The objective weight vector of evaluation index of coal supplier calculated by entropy method is $w^{\prime \prime}=\left(w_{1}^{\prime \prime}, w_{2}^{\prime \prime}, \ldots, w_{n}^{\prime \prime}\right)^{T}$, which satisfis $w_{j}^{\prime \prime} \in[0,1], \quad \sum_{j=1} w_{j}^{\prime \prime}=1$. The final weight vector is obtained by weighting the subjective weight and objective weight:

$$
w=\alpha w^{\prime}+\beta w^{\prime \prime}
$$

Where $\quad \alpha, \beta \quad$ satisfy $\quad \alpha, \beta>0, \alpha+\beta=1$, $\alpha, \beta>0, \alpha+\beta=1$

In order to fully reflect the subjective information and objective information in the scheme ranking, this paper starts from the weighted attribute value, considers that the weighted attribute value determined by the subjective weight tends to be consistent with the weighted attribute value determined by the objective weight, and establishes 
the optimization model of the coefficient $\alpha, \beta$ in the combination weight. According to formula (5-25), the subjective weighted attribute value of scheme ${ }^{a_{i}}$ under attribute $u_{j}$ is $r_{i j} \alpha w_{j}$, the objective weighted attribute value is $r_{i j} \beta w_{j}^{\prime}$, and the difference between subjective and objective weighted attribute values is $r_{i j} \alpha w_{j}-r_{i j} \beta w_{j}^{\prime}$. Therefore, the deviation degree of subjective and objective decision information of scheme $a_{i}$ is given as follows:

$$
d_{i}=r_{i j} \alpha_{j} w_{j}-r_{i j} \beta_{j} w_{j}^{\prime}
$$

Obviously, the smaller $d_{i}$ is, the more consistent the subjective and objective decision information of scheme $a_{i}$ is. Therefore, the following optimization model is constructed as follows:

$$
\min D=\left(d_{1}, d_{2}, \ldots, d_{m}\right)
$$

Obviously, it is a multi-objective decision programming problem. Since there is fair competition among the alternatives and there is no preference relationship, the above multi-objective programming model can be transformed into the following equivalent single objective programming model by the equal weight linear weight sum method.

$$
\begin{gathered}
\min Z=\sum_{i=1}^{m} d_{i}=\sum_{i=1}^{m} \sum_{j}^{n}\left(r_{i j} \alpha_{j} w_{j}-r_{i j} \beta_{j} w_{j}^{\prime}\right) \\
\text { s.t. } \alpha_{j}+\beta_{j}=1\left(\alpha_{j}, \beta_{j} \geq 0\right)
\end{gathered}
$$

\section{Comprehensive benefit evaluation model of virtual power plants considering electric vehicles}

The traditional evaluation methods mainly include ELECTRE method, ideal point method and PROMETHEE method. Different methods have different application scope. In the process of evaluation, information omission and unreasonable index standardization will lead to the incompatibility of individual evaluation results and low credibility of evaluation results. In this section, the ideal point method is applied to improve the matter-element extension model, and the ideal matter-element extension evaluation model of virtual power plants benefit is established.

\subsection{Matter-element extension evaluation}

The idea of the matter-element extension evaluation method is given as follows: firstly, according to the existing data, the level of the evaluation object is divided into several levels, and the data range of each level is given by the database or expert opinions. Then, the indexes of the evaluation object are substituted into the set of each level for multi-index evaluation. The evaluation results are compared according to the correlation degree between the evaluation object and the set of each level. The larger the correlation degree is, the better the consistency between it and its grade set will be.

Suppose that $N$ has characteristic $c$ and its value is $\mathrm{V}$, then the ordered triple $R=(N, c, v)$ composed of $N 、 c$ 、 $v$ is regarded as the basic element to describe things. Suppose that $N$ has multiple features, which can be described by $n$ features $c_{1}, c_{2}, \cdots, c_{n}$ and corresponding quantity $v_{1}, v_{2}, \cdots, v_{n}$, then matter-element $R$ is called $n$-dimensional matter-element, which is given as follows:

$$
R=(N, C, V)=\left[\begin{array}{c}
R_{1} \\
R_{2} \\
\vdots \\
R_{n}
\end{array}\right]=\left[\begin{array}{ccc}
N & c_{1} & v_{1} \\
& c_{2} & v_{2} \\
& \vdots & \vdots \\
& c_{n} & v_{n}
\end{array}\right]
$$

Where $R_{i}=\left(N_{i}, C_{i}, V_{i}\right), i=1,2, \ldots, n$ represents the fractional matter-element of $\mathrm{R}, C=\left[c_{1}, c_{2}, \ldots, c_{n}\right]$ represents the eigenvector, and $V=\left[v_{1}, v_{2}, \ldots, v_{n}\right]$ represents the magnitude of the eigenvector.

The specific evaluation steps are given as follows: firstly, the matter-element formed by classical domain, node domain and object to be identified is determined.

$$
R_{j}=\left(N_{j}, C_{i}, V_{j i}\right)=\left[\begin{array}{ccc}
N_{j} & c_{1} & v_{j 1} \\
& c_{2} & v_{j 2} \\
& \vdots & \vdots \\
& c_{n} & v_{j n}
\end{array}\right]
$$

Where $N_{j}$ represents the j grades, $c_{1}, c_{2}, \ldots, c_{n}$ is the $\mathrm{n}$ different characteristics of $N_{j}$, and $v_{j 1}, v_{j 2}, \ldots, v_{j n}$ is the range of $N_{j}$ values about $c_{1}, c_{2}, \ldots, c_{n}$, which is the classical domain.

$$
R_{p}=\left(p, C_{i}, V_{p i}\right)=\left[\begin{array}{ccc}
p & c_{1} & \left\langle a_{p 1}, b_{p 1}\right\rangle \\
& c_{2} & \left\langle a_{p 2}, b_{p 2}\right\rangle \\
\vdots & \vdots \\
& c_{n} & \left\langle a_{p n}, b_{p n}\right\rangle
\end{array}\right]
$$

Where $p$ represents the whole class of the object to be evaluated; $v_{p 1}, v_{p 2}, \ldots, v_{p n}$ represent the range of $p$ value with respect to $c_{1}, c_{2}, \ldots, c_{n}$, that is, the node domain of $p$.

$$
R_{0}=\left[\begin{array}{ccc}
p_{0} & c_{1} & v_{1} \\
& c_{2} & v_{2} \\
& \vdots & \vdots \\
& c_{n} & v_{n}
\end{array}\right]
$$


Where $p_{0}$ represents the material element to be evaluated, $v_{1}, v_{2}, \ldots, v_{n}$ represent the specific data of $p_{0}$ about $c_{1}, c_{2}, \ldots, c_{n}$.

Then, the evaluation element is normalized. When the measured value of the evaluation index exceeds the node domain, the correlation function will be unable to calculate. At this time, the matter-element extension method cannot be used to evaluate the power generation performance. In order to overcome this limitation, this section normalizes the quantity value of each classical domain and the matter-element to be evaluated on the basis of the original matter-element extension method, that is, divide them by the right end value $b_{p i}$ of the node domain $V_{p}$ to get a new matter-element classical domain The specific calculation is given as follows:

$$
R_{j}^{\prime}=\left(N_{j}, C_{i}, V_{j i}^{\prime}\right)=\left[\begin{array}{ccc}
N_{j} & c_{1} & <\frac{a_{j 1}}{b_{p 1}}, \frac{b_{j 1}}{b_{p 1}}> \\
c_{2} & <\frac{a_{j 2}}{b_{p 2}}, \frac{b_{j 2}}{b_{p 2}}> \\
\vdots & \vdots \\
c_{n} & <\frac{a_{j n}}{b_{p n}}, \frac{b_{j n}}{b_{p n}}>
\end{array}\right]
$$

Finally, the correlation degree between the object to be evaluated and the matter-element is calculated, and the evaluation results are compared according to the correlation degree between it and each grade set. The larger the correlation degree is, the better the consistency between it and its grade set is.

$$
D\left(v, V_{j i}^{\prime}\right)=\left|v-\frac{a+b}{2}\right|-\frac{b-a}{2}
$$

Where $v$ represents the point value; $a, b$ represent the left and right end values of the interval respectively, and the calculation of correlation degree is as given follows:

$$
\begin{aligned}
& \qquad K_{j}\left(p_{0}\right)=1-\sum_{i=1}^{n} w_{i} D_{i j} \\
& \text { If } K_{j}\left(p_{0}\right)=\max \left\{K_{j}\left(p_{0}\right)\right\}(j=1,2, \ldots, \mathrm{m}) \text {, then } \\
& p_{0} \text { belongs to } j \text { grade. } \\
& 3.2 \text { Ideal matter-element extension evaluation } \\
& \text { model }
\end{aligned}
$$

\subsection{Ideal matter-element extension evaluation model}

The reliability of the traditional matter-element evaluation results mainly depends on the rationality of the classical domain and node domain. When the classical domain is set unreasonably, the evaluation objects may be in the same evaluation level, and only part of the ranking of the evaluation scheme can be obtained. In order to overcome this problem, this paper uses the basic idea of ideal point method to improve the traditional matter-element extension model. The extension model of ideal matterelement is constructed by setting positive and negative ideal matter-element to replace the traditional classical domain and measuring the distance between the matterelement to be evaluated and the positive and negative ideal matter-element by using the closeness degree to replace the maximum membership criterion. In general, the selection of positive and negative ideal matter-element can be based on expert judgment, or the best and worst evaluation index can be selected from the matter-element to be evaluated to form positive and negative ideal matterelement.

The determination of positive and negative ideal matter-element can be based on experts' opinions, or the best (or worst) data can be selected from each index of matter-element to be evaluated as the positive (negative) ideal matter-element. If index $c_{i}$ is a positive index, then $v_{j i}^{+}=\max \left(v_{i}\right) ;$ if index $c_{i}$ is a negative index, then $v_{j i}^{+}=\min \left(v_{i}\right)$; if index $c_{i}$ is an intermediate index (the smaller the difference with the standard deviation, the better), then $v_{j i}^{+}=\sigma\left(v_{i}\right)$; if index $c_{i}$ is an interval index (stable within the interval $\left[u_{1 i}, u_{2 i}\right]$, the best), then $v_{j i}^{+}$takes the interval value $v_{j i}^{+}=\left[u_{1 i}, u_{2 i}\right]$. Then the positive ideal matter-element $R^{+}$can be obtained. Similarly, negative ideal matter-element $R^{-}$can be obtained.

The closeness refers to the degree that the evaluated sample and the standard sample are close to each other. The larger the value is, the closer they are. Otherwise, they are far away from each other. Therefore, we can directly judge the merits of each evaluation object according to the closeness to the positive and negative ideal elements, and can also divide the positive and negative ideal elements into different levels, and calculate the closeness to each level to determine the merits of the location. In this section, the asymmetric closeness is selected as the closeness function, and the value of closeness function is calculated, which is given as follows:

$$
N=1-\frac{1}{n(n+1)} \sum_{i=1}^{n} D w_{i}
$$

Where $N$ is the closeness ; $D$ is the distance; $w_{i}$ is the weight, then the closeness of the matter-element to be evaluated corresponding to each level is given as follows:

$$
N_{j}\left(p_{0}\right)=1-\frac{1}{n(n+1)} \sum_{i=1}^{n} D_{j}\left(v_{i}^{\prime}\right) w_{i}(X)
$$


Where $D_{j}\left(v_{i}^{\prime}\right)$ is the closeness between the evaluated matter-element $R_{0}$ and the normalized ideal matterelement classical domain, the calculation formula is given as follows:

$$
\begin{aligned}
& D_{j}^{+}\left(v_{i}^{\prime}\right)=\left|v_{i}^{\prime}-v_{j i}^{+}\right| \\
& D_{j}^{-}\left(v_{i}^{\prime}\right)=\left|v_{i}^{\prime}-v_{j i}^{-}\right|
\end{aligned}
$$

The closeness between the matter-element to be evaluated and the positive and negative ideal matterelement is given as follows

$$
\begin{aligned}
& N_{j}^{+}\left(p_{0}\right)=1-\frac{1}{n(n+1)} \sum_{i=1}^{n}\left|v_{i}^{\prime}-v_{j i}^{+}\right| w_{i}(X) \\
& N_{j}^{-}\left(p_{0}\right)=1-\frac{1}{n(n+1)} \sum_{i=1}^{n}\left|v_{i}^{\prime}-v_{j i}^{-}\right| w_{i}(X)
\end{aligned}
$$

The comprehensive closeness is given as follows:

$$
\bar{N}_{j}\left(p_{0}\right)=\frac{N_{j}^{+}\left(p_{0}\right)}{N_{j}^{+}\left(p_{0}\right)+N_{j}^{-}\left(p_{0}\right)}
$$

\subsection{Model fitness analysis}

In order to judge the advantages and disadvantages of the evaluation results of different methods, this paper selects the compatibility degree and difference degree to evaluate the representativeness of the evaluation methods. If the degree of compatibility is large, the representativeness of the scheme is strong and the reliability is high. The difference degree refers to taking the ranking of evaluation schemes as the benchmark value, and the smaller the difference degree is, the better the evaluation method is.

The compatibility of each evaluation method is as given follows:

$$
\begin{gathered}
r_{i j}=1-\frac{6}{n\left(n^{2}-1\right)} \sum_{k=1}^{n}\left(a_{k}^{(i)}-a_{k}^{(j)}\right)^{2} \\
r_{z}=\sum_{j=1}^{h-1} w_{j} r_{i j}
\end{gathered}
$$

Where $a_{k}^{(i)}$ and $a_{k}^{(j)}$ respectively represent the ranking order of evaluation index $k$ in evaluation method $i$ and evaluation method $j, i, j=1,2, \ldots h ; \mathrm{n}$ is the total number of evaluation indexes; $h$ is the total number of evaluation methods. In general, when there is no special preference for the evaluation scheme, $w_{j}=1 /(h-1)$;

$r_{z}$ represents the compatibility of the evaluation scheme. The difference degree of each evaluation scheme is given as follows:

$$
d_{z}=\frac{1}{h-1} \sum_{j=1}^{h} d_{z j}, i, j=1,2, \ldots, h
$$

Where $d_{z}$ represents the difference degree between a certain evaluation scheme and other h-levaluation schemes; $d_{z j}$ represents the number of evaluation objects in a certain number range of the evaluation scheme that exceed the specified number range in the $\mathrm{j}$ evaluation scheme.

\section{Example analysis}

\subsection{Basic data}

In order to analyze the comprehensive benefit of different virtual power plants, this paper sets four kinds of virtual power plants as evaluation schemes, which are shown in Table $2^{8}$.

Table 2. Four evaluation schemes of virtual power plants.

\begin{tabular}{ccccc}
\hline & Scheme & Scheme & Scheme & Scheme \\
& 1 & 2 & 3 & 4 \\
\hline Wind turbine & $\sqrt{ }$ & $\sqrt{ }$ & $\sqrt{ }$ & $\sqrt{ }$ \\
Photovoltaic & $\sqrt{ }$ & $\sqrt{ }$ & $\sqrt{ }$ & $\sqrt{ }$ \\
unit & & & & \\
$\begin{array}{c}\text { Energy storage } \\
\text { battery }\end{array}$ & $\sqrt{ }$ & $\sqrt{ }$ & $\sqrt{ }$ & $\sqrt{ }$ \\
$\begin{array}{c}\text { Gas turbine } \\
\text { Interruptible } \\
\text { load }\end{array}$ & $\sqrt[V]{ }$ & $\sqrt{ }$ & $\sqrt{ }$ & $\sqrt{ }$ \\
$\begin{array}{c}\text { Proportion of } \\
\text { electric vehicles }\end{array}$ & $0 \%$ & $80 \%$ & $100 \%$ & 120 \\
\hline
\end{tabular}

The " $\sqrt{ }$ " in the table represent that the equipment is included in the virtual power plant. The number of electric

\begin{tabular}{|c|c|c|c|c|c|c|}
\hline \multirow{2}{*}{ Index } & \multirow{2}{*}{ Unit } & \multirow{2}{*}{ Type } & \multicolumn{4}{|c|}{ Different virtual power plant schemes } \\
\hline & & & Scheme 1 & Scheme 2 & Scheme 3 & Scheme 4 \\
\hline $\mathrm{C} 1$ & yuan $/ \mathrm{kW}$ & Positive & 3150 & 2200 & 1200 & 2600 \\
\hline $\mathrm{C} 2$ & yuan//MW·h & Positive & 205 & 210 & 260 & 225 \\
\hline $\mathrm{C} 3$ & yuan//MW·h & Positive & 35 & 50 & 120 & 80 \\
\hline $\mathrm{C} 4$ & yuan $/ / \mathrm{kW}$ & Positive & 3050 & 2600 & 1500 & 2100 \\
\hline $\mathrm{C} 5$ & - & Positive & high & moderate & common & moderate \\
\hline
\end{tabular}
vehicles in a province in 2020 is 210100 . If the proportion of electric vehicles is $80 \%$, it means $80 \%$ of 210100 , that is 168100 . The qualitative indexes are assigned in the form of questionnaire, and the quantitative indexes are calculated by formula to get the initial assignment results of different indexes, which is shown in Table 3

Table 3. Initial value of benefit evaluation index of different virtual power plant schemes. 


\begin{tabular}{lcccccc}
\hline C6 & - & Positive & common & high & moderate & high \\
C7 & 100 million yuan & Negative & 0.82 & 1.48 & 2.85 & 1.76 \\
C8 & $\%$ & Positive & 10.28 & 13.65 & 18.26 & 14.82 \\
C9 & - & Negative & moderate & lower & low & lower \\
C10 & ten thousand yuan & Positive & 48.55 & 64.85 & 79.44 & 66.73 \\
C11 & yuan/person & Negative & 1185.12 & 2258.45 & 3245.64 & 1625.48 \\
C12 & ton & Positive & 115.08 & 130.18 & 180.37 & 140.25 \\
C13 & kg & Positive & 373.35 & 422.34 & 585.18 & 455.01 \\
C14 & kg & Positive & 325.04 & 367.68 & 509.45 & 396.13 \\
C15 & - & Positive & moderate & higher & high & high \\
C16 & ten thousand yuan & Negative & 2.26 & 2.55 & 3.54 & 2.75 \\
C17 & $\%$ & Positive & 75 & 80 & 90 & 80 \\
C18 & $\%$ & Negative & 65 & 54 & 38 & 49 \\
C19 & $\%$ & Positive & 75 & 80 & 90 & 80 \\
C20 & - & Positive & moderate & higher & high & higher \\
C21 & $\%$ & Positive & 12.34 & 16.85 & 19.18 & 18.24 \\
C22 & - & Positive & moderate & higher & high & higher \\
C23 & - & Positive & common & moderate & high & moderate \\
C24 & - & Positive & common & moderate & high & moderate \\
\hline
\end{tabular}

\subsection{Result analysis}

evaluation indexes of the virtual power plants are calculated, which is shown in Table 4.

The comprehensive weight of the comprehensive benefit

Table 4. Simplified indexes and weight calculation for comprehensive benefit evaluation of virtual power plants.

\begin{tabular}{|c|c|c|c|c|c|c|}
\hline \multirow{2}{*}{ Index } & \multirow{2}{*}{ New number } & \multicolumn{3}{|c|}{ ANP method } & \multirow{2}{*}{$\begin{array}{l}\text { Entropy weighting } \\
\text { method }\end{array}$} & \multirow{2}{*}{$\begin{array}{c}\text { Integrated weighting } \\
\text { method }\end{array}$} \\
\hline & & Local weight & Limit relative weight & Weight & & \\
\hline $\mathrm{C} 1$ & D1 & 0.474 & 0.079 & 0.09 & 0.14 & 0.13 \\
\hline $\mathrm{C} 2$ & D2 & 0.253 & 0.043 & 0.038 & 0.068 & 0.062 \\
\hline $\mathrm{C} 3$ & D3 & 0.139 & 0.024 & 0.117 & 0.038 & 0.052 \\
\hline $\mathrm{C} 5$ & D4 & 0.080 & 0.014 & 0.077 & 0.022 & 0.031 \\
\hline $\mathrm{C} 7$ & D5 & 0.043 & 0.007 & 0.068 & 0.012 & 0.019 \\
\hline $\mathrm{C} 8$ & D6 & 0.686 & 0.159 & 0.040 & 0.256 & 0.235 \\
\hline $\begin{array}{l}\mathrm{C} \\
10\end{array}$ & D7 & 0.189 & 0.044 & 0.038 & 0.070 & 0.064 \\
\hline $\begin{array}{c}\mathrm{C} \\
11\end{array}$ & D8 & 0.038 & 0.009 & 0.056 & 0.014 & 0.021 \\
\hline $\begin{array}{c}\mathrm{C} \\
13\end{array}$ & D9 & 0.018 & 0.019 & 0.196 & 0.029 & 0.046 \\
\hline $\begin{array}{l}\mathrm{C} \\
14\end{array}$ & D10 & 0.814 & 0.046 & 0.037 & 0.073 & 0.067 \\
\hline $\begin{array}{l}\mathrm{C} \\
15\end{array}$ & D11 & 0.176 & 0.010 & 0.050 & 0.016 & 0.022 \\
\hline $\begin{array}{c}\mathrm{C} \\
19\end{array}$ & D12 & 0.188 & 0.102 & 0.043 & 0.165 & 0.152 \\
\hline $\begin{array}{c}\mathrm{C} \\
20\end{array}$ & D13 & 0.056 & 0.102 & 0.056 & 0.049 & 0.048 \\
\hline $\begin{array}{l}\mathrm{C} \\
21\end{array}$ & D14 & 0.009 & 0.005 & 0.053 & 0.008 & 0.013 \\
\hline $\begin{array}{c}\mathrm{C} \\
22\end{array}$ & D15 & 0.045 & 0.025 & 0.041 & 0.040 & 0.038 \\
\hline
\end{tabular}

Then, Equations (1) to (3) are applied to standardize the benefit evaluation indexes. Furthermore, the traditional matter-element extension evaluation model and the ideal matter-element extension evaluation model are applied to the comprehensive evaluation of the virtual power plants respectively. The application steps of the traditional matter-element extension evaluation model are given as follows:

(1) Establish classical domain and node domain

The comprehensive benefit of the virtual power plants are divided into five levels, namely excellent, good, medium, qualified and unqualified. The classical domain of each evaluation index is obtained by questionnaire from experts, and the values of the standardized evaluation 
indexes are distributed between [0,1]. Fig. 1 shows the classical domain and node domain.

\begin{tabular}{|c|c|c|c|c|c|c|c|c|c|c|c|}
\hline$\left[\begin{array}{ll}N_{1} & D\end{array}\right]$ & $(0.6,1)]$ & {$\left[\begin{array}{ll}N_{2} & D_{1}\end{array}\right.$} & $P_{1} \quad(0.4,0.6)$ & {$\left[\begin{array}{ll}N_{3} & D_{1}\end{array}\right.$} & $(0.3,0.4)]$ & $\left\lceil\begin{array}{ll}N_{1} & D\end{array}\right.$ & $P_{1}(0.2,0.3)$ & {$\left[\begin{array}{ll}N_{5} & D_{1}\end{array}\right.$} & $\begin{array}{ll}D_{1} & (0,0.2)\end{array}$ & $\left\lceil N_{p}\right.$ & $\left.D_{1} \quad(0,1)\right]$ \\
\hline$D$ & $(0.8,1)$ & $D_{2}$ & $D_{2}(0.6,0.8)$ & $D_{2}$ & $(0.4,0.9)$ & $D_{2}$ & $2 \quad(0.1,0.4)$ & & $D_{2} \quad(0,0.1)$ & & $D_{2} \quad(0,1)$ \\
\hline$D$ & $(0.8,1)$ & $D_{3}$ & $D_{3}(0.5,0.8)$ & $D_{3}$ & $(0.3,0.5)$ & $D_{1}$ & $g_{3}(0.2,0.3)$ & & $D_{3} \quad(0,0.2)$ & & $D_{3}(0,1)$ \\
\hline & $(0.7,1)$ & $D_{4}$ & $7_{4}(0.5,0.7)$ & $D_{4}$ & $(0.3,0.5)$ & $D_{4}$ & $(0.1,0.3)$ & & $D_{4} \quad(0,0.1)$ & & $D_{4} \quad(0,1)$ \\
\hline & $(0.8,1)$ & $D_{\mathrm{g}}$ & $D_{S}(0.6,0.8)$ & $D_{s}$ & $(0.3,0.6)$ & $D_{3}$ & $g_{5} \quad(0.1,0.3)$ & & $D_{5} \quad(0,0.1)$ & & $D_{5}(0,1)$ \\
\hline D & $(0.5,1)$ & $D_{\mathrm{a}}$ & $D_{6}(0.4,0.5)$ & $D_{6}$ & $(0.3,0.4)$ & $D_{\mathrm{a}}$ & $D_{6}(0.2,0.3)$ & & $D_{6} \quad(0,0.2)$ & & $D_{6} \quad(0,1)$ \\
\hline$D$ & $(0.7,1)$ & $D_{3}$ & $(0.5,0.7)$ & $D_{4}$ & $(0.3,0.5)$ & $D_{3}$ & $2 \quad(0.1,0.3)$ & & $D_{7} \quad(0,0.1)$ & & $D_{7}(0,1)$ \\
\hline & $(0.8,1) R_{2}$ & $D_{k}$ & $\mathcal{S}_{8}(0.5,0.8) R$ & $D_{8}$ & $(0.3,0.5) \mid R_{3}$ & $D_{8}$ & $S_{8}(0.2,0.3) K$ & & $D_{\mathrm{s}}(0,0.2)$ & & $D_{\mathrm{g}}(0,1)$ \\
\hline$L$ & $(0.7,1)$ & $D_{3}$ & $D_{9} \quad(0.5,0.7)$ & $D_{9}$ & $(0.4,0.6)$ & $D_{9}$ & $D_{9} \quad(0.1,0.3)$ & & $D_{9}(0,0.1)$ & & $D_{9}(0,1)$ \\
\hline & $(0.8,1)$ & $D_{x}$ & (100 $(0.6,0.8)$ & $D_{\text {vi }}$ & $(0.4,0.6)$ & $D_{10}$ & $R_{10}(0.2,0.4)$ & & $D_{10}(0,0.2)$ & & $D_{10}(0,1)$ \\
\hline & $(0.8,1)$ & $D_{1}$ & $D_{11}(0.6,0.8)$ & $D_{11}$ & $(0.4,0.6)$ & $D_{11}$ & $b_{11}(0.1,0.4)$ & & $D_{11}(0,0.1)$ & & $D_{11}(0,1)$ \\
\hline D & $(0.7,1)$ & $D_{I_{1}}$ & $p_{12}(0.5,0.7)$ & $D_{12}$ & $(0.3,0.5)$ & $D_{2}$ & $p_{12}(0.1,0.3)$ & & $D_{12}(0,0.1)$ & & $D_{12}(0,1)$ \\
\hline D & $(0.5,1)$ & $D_{1}$ & ${ }_{13}(0.4,0.5)$ & $D_{B}$ & $(0.3,0.4)$ & $D_{\mathrm{B}}$ & $D_{13}(0.2,0.3)$ & & $D_{13}(0,0.2)$ & & $D_{13}$ \\
\hline D & $(0.6,1)$ & $D_{x}$ & 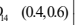 & $D_{4}$ & $(0.3,0.4)$ & $D_{4}$ & 14 $(0.2,0.3)$ & & $D_{14}(0,0.4)$ & & $D_{14}(0,1)$ \\
\hline & $=(0.8,1)]$ & $D_{1}$ & 15 & $D_{s}$ & $(0.3,0.6]$ & $D_{\mathrm{s}}$ & $\left.7_{15}(0.1,0.3)\right]$ & & $\left.D_{15}(0.8,1)\right]$ & & $\left.D_{15}(0,1)\right]$ \\
\hline
\end{tabular}

(2) Determine the relevance between the object to be evaluated and the classical domain

Equations (1) to (3) are applied to evaluate the comprehensive benefit of virtual power plants, and the relevance between four kinds of virtual power plants and classical domain is obtained, which is shown in Table 5.

Fig. 1. Classical domain and node domain.

Table 5. Evaluation results of the traditional matter-element extension model.

\begin{tabular}{ccccccc}
\hline Evaluation object & Excellent & Good & Secondary & Medium & Unqualified & Grade \\
\hline Scheme 1 & 0.9823 & 0.9818 & 0.9831 & 0.9827 & 0.9830 & Medium \\
Scheme 2 & 0.9840 & 0.9844 & 0.9835 & 0.9832 & 0.9813 & Good \\
Scheme 3 & 0.9872 & 0.9851 & 0.9835 & 0.9820 & 0.9790 & Excellent \\
Scheme 4 & 0.9841 & 0.9835 & 0.9835 & 0.9823 & 0.9804 & Excellent \\
\hline
\end{tabular}

According to Table 5, the comprehensive correlation degree of scheme 1 is [0.9823, 0.9818, 0.9831, 0.9827, 9830]. it can be seen from the size of each relevance that the evaluation grade of scheme 1 belongs to "medium", while the evaluation grades of scheme 2 , scheme 3 and scheme 4 belong to "good", "excellent" and "excellent" respectively. The ranking relationship of different clean energy integrated consumption channels is represented by the level relationship of PROMETHEE method, which is shown in Fig. 2.

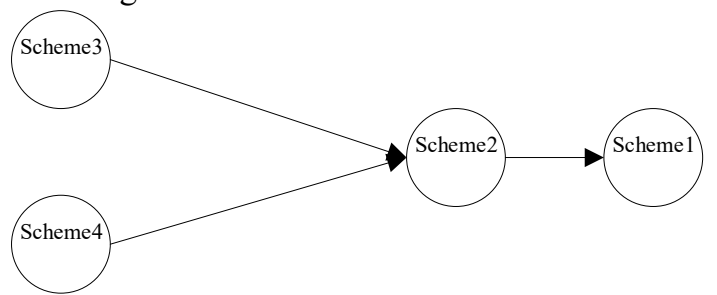

Fig. 2. Evaluation results of traditional matter-element extension model.

According to Fig 2, it can be seen that the traditional matter-element extension evaluation model can be used to evaluate the comprehensive benefit of virtual power plants, and can obtain the overall ranking of advantages and disadvantages. However, there are some local ranking problems that cannot be accurately evaluated. For example, the evaluation grades of scheme 3 and scheme 4 are "excellent", but there is no way to distinguish which is better. Therefore, this paper introduces the ideal point method to improve the traditional matter-element extension evaluation model, and selects positive and negative ideal matter-element $R^{+}$and $R^{-}$ respectively to evaluate the comprehensive benefit of virtual power plants.

(3) Calculate comprehensive closeness

Equations (30) to (31) are used to calculate the closeness degree and comprehensive closeness degree between the object to be evaluated and the positive and negative ideal matter-elements, and the comprehensive benefit evaluation results of the virtual power plant are obtained. Table 6 shows the comprehensive closeness of the matter-element to be evaluated.

Table 6. The comprehensive closeness of the matter-element to be evaluated.

\begin{tabular}{ccccc}
\hline & $N_{j}^{+}\left(p_{0}\right)$ & $N_{j}^{-}\left(p_{0}\right)$ & $N_{j}\left(p_{0}\right)$ & Ranking \\
\hline $\begin{array}{c}\text { Scheme } \\
1\end{array}$ & 0.9808 & 0.9861 & 0.4937 & 4 \\
$\begin{array}{c}\text { Scheme } \\
2\end{array}$ & 0.9857 & 0.9812 & 0.4961 & 3 \\
$\begin{array}{c}\text { Scheme } \\
3\end{array}$ & 0.9879 & 0.9790 & 0.4972 & 1 \\
$\begin{array}{c}\text { Scheme } \\
4\end{array}$ & 0.9876 & 0.9793 & 0.4971 & 2 \\
\hline
\end{tabular}

According to Table 6, it can be seen that the ranking of scheme 1 and scheme 2 is consistent with the result of traditional matter-element extension evaluation model. At the same time, the ranking of scheme 3 and scheme 4 has been clearly obtained, that is, the evaluation result of multi energy complementary system is better than that of virtual power plant, which is shown in Fig. 3.

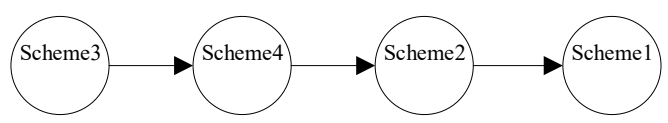

Fig. 3. Evaluation results of ideal matter-element extension model.

According to the above evaluation results, for the four kinds of virtual power plants considered in this paper, when the electric vehicle access ratio reaches $120 \%$, the comprehensive income is the highest. This is mainly because the electric vehicles connected to the virtual 
power plants has the dual properties of energy storage and power generation through reasonable and orderly charging and discharging. On the one hand, it can improve the consumption capacity of wind power and photovoltaic power; on the other hand, it can realize the environmentfriendly characteristics of the system by replacing traditional fossil energy with clean energy. In general, actively guiding electric vehicles to access virtual power plants and promoting the integrated and optimized utilization of clean energy are conducive to giving full play to the economic benefit and environmental benefit of clean energy and achieving the best overall benefit of the system.

\section{Conclusions}

The benefit of different types of virtual power plants considering electric vehicles is analyzed and evaluated in this paper. When the access proportion of electric vehicles reaches $120 \%$, the comprehensive benefit of virtual power plants is the highest. Therefore, in the follow-up development process of virtual power plants, it is necessary to actively guide electric vehicles to join virtual power plants to promote the integrated and optimized utilization of clean energy.

\section{Acknowledgment}

This work is supported by Science and Technology Project of State Grid Corporation of China. (Research on market mechanism and business model of virtual power plant under the background of energy Internet; No.: 1400202057442A-0-0-00).

\section{References}

1. Wang Ruizhuo, Liu huaidong, Cui Liyao, Zhao Yanqi, Liu Hao. Member selection model of virtual power plant for thermal wind storage high load energy enterprise considering comprehensive efficiency evaluation [J / OL]. Power grid technology: 1-9 [2021-02-01] http://kns.cnki.net/kcms/detail/11.2410.TM.2020081 0.0944.002.html.

2. Li Wei, Yan Ning, Zhang Bo, et al. Economic dispatch of energy storage system in wind farm based on scenario analysis [J]. New electrical energy technology, 2018,37 (8): 40-47

3. Yan Liping, Liu Rui, Lu Xiaolan. Study on assessment and evaluation of grid connection of renewable energy power stations [J]. Qinghai electric power, 2014,33 (1): 45-47

4. Xu Jiamin, sun Yingyun, sun Qian, Ma Jianwei. Comprehensive evaluation index system for active power dispatching of virtual power plant $[\mathrm{J}]$. New electrical energy technology, 2020,39 (03): 32-39

5. Pan Ersheng, Wang Xinlei, Xu Tong, et al. Technology and Practice for promoting renewable energy power acceptance $[\mathrm{J}]$. Electric power construction, 2017,38 (2): 1-11

6. $\mathrm{Xu}$ Jiamin. Research on comprehensive evaluation technology of virtual power plant participating in active power coordination and optimal dispatch of power grid [D]. North China Electric Power University (Beijing), 2019

7. Shekarian E, Kazemi N, Abdul-Rashid S H, et al. Fuzzy inventory models: A comprehensive review [J]. Applied Soft Computing, 2017,55:588-621.

8. Kong Xiangyu,Xiao Jie,Liu Dehong,Wu Jianzhong,Wang Chengshan,Shen Yu. Robust stochastic optimal dispatching method of multienergy virtual power plant considering multiple uncertainties[J]. Applied Energy,2020,279. 